\title{
Effect of Food on the Pharmacokinetics of Desvenlafaxine in Healthy Subjects
}

\author{
Alice I Nichols ${ }^{1 *}$, Lyette S Richards ${ }^{1}$, Jessica Behrle ${ }^{2}$, Joel A Posener ${ }^{3}$ and Jeffrey Paul ${ }^{4}$ \\ ${ }^{1}$ Pfizer Inc, formerly Wyeth Research, Collegeville, Pennsylvania, USA \\ ${ }^{2}$ Springhouse, Pennsylvania, USA \\ ${ }^{3}$ Philadelphia, Pennsylvania, USA
}

${ }^{4}$ Deerfield, Illinois, USA

\begin{abstract}
The serotonin-norepinephrine reuptake inhibitor desvenlafaxine has linear and dose-proportional pharmacokinetics from 25 to $900 \mathrm{mg}$. The effect of diet on the pharmacokinetics, safety, and tolerability of desvenlafaxine in healthy volunteers $(\mathrm{N}=33)$ was assessed in this single-dose, open-label, randomized, 4-period, 4-sequence, crossover, inpatient study. Desvenlafaxine $200 \mathrm{mg}$ was administered after an overnight fast or after a low-, medium-, or high-fat breakfast. Blood samples were obtained over 72 hours. Pharmacokinetic parameters were compared across dosing conditions using analysis of variance. The median time to peak concentration for desvenlafaxine was approximately 6 hours under fasting conditions and was delayed by approximately 2 to 4 hours when administered with food. Diet did not affect apparent oral-dose clearance and apparent terminal-phase elimination half-life values. Except for peak plasma concentration under high-fat conditions, both peak plasma concentration and area under the plasma concentration-versus-time curve met bioequivalence test criteria under each dietary condition, compared with an overnight fast (90\% confidence interval within $80 \%-125 \%$ limits). The minor increase in peak plasma concentration under high-fat conditions was not clinically relevant. Desvenlafaxine was well tolerated under fasting and fed conditions. These results suggest that pharmacokinetics should not be a factor when considering whether to administer desvenlafaxine with or without food.
\end{abstract}

Keywords: Diet; Pharmacokinetics; Safety; Bioavailability; Desvenlafaxine

Abbreviations: 5-HT: Reuptake of Serotonin; AE: Adverse Event; ANOVA: Analysis of Variance; CYP450: cytochrome P450; AUC: Area Under the Plasma Concentration-Versus-Time Curve; $\mathrm{AUC}_{\mathrm{T}}$ : AUC to the Last Quantifiable Concentration at Time T; $\mathrm{Cl} / \mathrm{F}$ : Oral-dose Clearance; $\mathrm{C}_{\max }$ : Peak Plasma Concentration; ECG: 12Lead Electrocardiogram; MDD: Major Depressive Disorder; NE: Norepinephrine; ODV: O-Desmethylvenlafaxine; PERIOD: Treatment Period; SEQ: Sequence; SPE: Solid Phase Extraction; SUB: Subject; $t_{1 / 2}$ : Half-life; TEAE: Treatment-Emergent Adverse Events; $t_{\max }$ : Time to Peak Plasma Concentration; TRT: Treatment

\section{Introduction}

Desvenlafaxine is the major active metabolite of the serotoninnorepinephrine reuptake inhibitor venlafaxine [1]; the free base of desvenlafaxine is also referred to as $\mathrm{O}$-desmethylvenlafaxine (ODV). It is administered clinically as the succinate salt, formulated as an extendedrelease tablet for once-a-day oral administration. Desvenlafaxine is approved for the treatment of major depressive disorder (MDD) [2]. Like venlafaxine, desvenlafaxine has little effect on dopamine reuptake and primarily inhibits the reuptake of serotonin (5-HT) and norepinephrine (NE) [1,3,4]. Desvenlafaxine has a unique selectivity ratio for the $5-\mathrm{HT}$ and $\mathrm{NE}$ transporters $[3,4]$; however, the clinical implications of this have not yet been determined. Desvenlafaxine has no monoamine oxidase activity and does not substantially interact with $\mathrm{H}_{1}$-histaminergic, muscarinic, or $\alpha_{1}$-adrenergic receptors [1,4].

Clinical trials in healthy volunteers have shown that desvenlafaxine is mainly eliminated unchanged by renal excretion, and that when it is metabolized, the process primarily involves phase II glucuronidation with renal excretion of the glucuronide metabolite [5]. Phase I hepatic metabolism of desvenlafaxine appears to play a small role in its elimination, marked by metabolism to $\mathrm{N}, \mathrm{O}$-didesmethylvenlafaxine by the cytochrome P450 (CYP450) 3A4 pathway [5]. Because desvenlafaxine is primarily metabolized via glucuronidation, and clinical reports have shown that desvenlafaxine exerts minimal effects on the activities of the CYP450 enzymes or p-glycoprotein, the potential ability of other drugs to alter desvenlafaxine metabolism is low [6-10].

Desvenlafaxine is a racemic mixture of the S- and R-enantiomers. Results from a prior study of absolute bioavailability showed approximately equal concentrations of the (R)- and (S)-enantiomers of desvenlafaxine from all plasma samples assayed, resulting in close agreement between pharmacokinetic parameters obtained for each enantiomer [11]. Due to the similarity between (R)- and (S)enantiomers of desvenlafaxine, desvenlafaxine pharmacokinetics are in general reported for the racemic mixture.

A study examining doses of 100, 300 and $600 \mathrm{mg}$ demonstrated that the pharmacokinetics of desvenlafaxine is linear and dose-proportional over the dose range of 100 to $600 \mathrm{mg}$ daily [12]. Additional investigation supports proportional decreases in exposure for doses of 25 and 50 $\mathrm{mg}$, in relation to a dose of $100 \mathrm{mg}$ (Data on file, Pfizer Inc, formerly Wyeth Research, Collegeville, PA). Small to moderate differences in pharmacokinetics, not considered to be clinically relevant, have been

*Corresponding author: Alice Nichols, PhD, Senior Director, Neuroscience Clinical Pharmacology, Wyeth Research, Collegeville, PA 19426, USA, Tel: (484) 865-8741; Fax: (484) 865-9075; E-mail: Alice.Nichols@pfizer.com

Received January 17, 2012; Accepted February 27, 2012; Published February 29,2012

Citation: Nichols Al, Richards LS, Behrle J, Posener JA, Paul J (2012) Effect of Food on the Pharmacokinetics of Desvenlafaxine in Healthy Subjects. J Bioequiv Availab 4: 024-029. doi:10.4172/jbb.1000106

Copyright: ( 2012 Nichols Al, et al. This is an open-access article distributed under the terms of the Creative Commons Attribution License, which permits unrestricted use, distribution, and reproduction in any medium, provided the original author and source are credited. 
observed between men and women with a $6 \%$ to $17 \%$ higher area under the plasma concentration-versus-time curve (AUC) in women compared to men when examined in 3 separate age groups (18-45 years of age; 65-75 years of age; and $>75$ years of age). Differences were partially explained by the smaller body weight in women compared to men (Data on file, Pfizer Inc, formerly Wyeth Research, Collegeville, $\mathrm{PA})$.

Clinical trials of desvenlafaxine administration in healthy adults [13] and in patients with MDD [14,15] have also demonstrated sustainedrelease characteristics that support once-daily administration. For clinical use, it is important to determine if the type of food intake affects pharmacokinetic behavior, as variability could result in unpredictable clinical effects and might necessitate specific dosing guidelines. Lack of variability would contribute to greater administration flexibility and patient convenience. The primary objective of the current study was to characterize the effect in healthy subjects of a low-, medium-, and high-fat meal (breakfast) on the pharmacokinetic profile of desvenlafaxine following a single, oral, $200 \mathrm{mg}$ dose. At the time the study was designed and conducted, $200 \mathrm{mg}$ was believed to be the upper range of therapeutic doses that would be available in clinical practice. However, the results of subsequent clinical trials $[14,15]$ demonstrated that the optimal dose was considerably lower, and $50 \mathrm{mg}$ is now the recommended daily therapeutic dose for MDD in the United States.

\section{Materials and Methods}

\section{Study design}

This was a single-dose, open-label, randomized, 4-period, 4-sequence, crossover, inpatient study. A randomized, crossover design was utilized in order to compare treatment effects within subjects using intrasubject variability rather than comparing subjects using the total variability (ie, intrasubject plus intersubject), thereby reducing the number of subjects required to attain the desired statistical power. Subjects were randomly assigned to treatment sequences consisting of an overnight fast (no breakfast), and low-, medium-, and high-fat breakfasts prior to receiving a single, oral dose of $200 \mathrm{mg}$ desvenlafaxine.

The study was carried out according to the Declaration of Helsinki in accordance with the principles of Good Clinical Practice. The protocols were reviewed and approved by an institutional review board. All subjects provided written, informed consent prior to enrollment.

\section{Study subjects}

Subjects were healthy males and females aged 18 to 45 years. Inclusion criteria at screening included nonpregnant, nonlactating women; body mass index between 18 and $31 \mathrm{~kg} / \mathrm{m}^{2}$ and body weight of at least $54 \mathrm{~kg}$; nonsmoker or smoker of fewer than 10 cigarettes per day, with the ability to abstain for 48 hours prior to, and throughout, the duration of the insubject confinement period; and abstinence from caffeine, alcohol, and grapefruit for 48 hours prior to, and through, 72 hours after the last test article administration. Exclusion criteria at screening included the presence or history of any significant disease and the use of any investigational or prescription drug within 30 days of study day -1 .

\section{Study procedures}

A prestudy screening evaluation was performed by a qualified staff member within 21 days of study day 1 , which included a physical examination, laboratory evaluation, vital sign measurement (supine blood pressure and pulse measured in duplicate after at least 10 minutes of rest, respiratory rate, and temperature), orthostatic evaluation (standing blood pressure and pulse performed at 1 and 3 minutes after the second set of vital sign measurements), and a standard 12-lead electrocardiogram (ECG).

Subjects were randomly assigned to a sequence of the following treatments: Treatment A: a single dose of desvenlafaxine $200 \mathrm{mg}$ under fasting conditions; Treatments B, C, D: a single dose of desvenlafaxine $200 \mathrm{mg}$ administered after completion of a low-fat breakfast, mediumfat breakfast, or high-fat breakfast, respectively. All subjects fasted overnight ( $\geq 10$ hours) prior to test article administration. Subjects assigned to the Treatment A group received desvenlafaxine the morning after the overnight fast (no breakfast); those assigned to Treatments $\mathrm{B}$ through $\mathrm{D}$ received desvenlafaxine 5 minutes after completion of the assigned breakfast. The fat content of the breakfasts was as follows: low fat, 16\% fat; medium fat, 30\% fat; and high fat, $\sim 55 \%$ fat. All subjects fasted for 4 hours following administration, after which time, standardized medium-fat meals and snacks were provided for the duration of the study period. Water was not permitted during the 2 hours before and 4 hours after desvenlafaxine administration. The final study evaluation included a physical examination, vital sign measurements, 12-lead ECG, and laboratory evaluations. A washout interval of at least 4 days separated each dose of desvenlafaxine. Study duration was approximately 38 days for each subject, including a $17-$ day (16-night) inpatient period.

\section{Blood sampling}

Venous blood samples were collected to measure concentrations of the (R)- and (S)-enantiomers of desvenlafaxine 1 day before the day of study and at $0.5,1,2,4,6,8,10,12,16,24,36,48,60$, and 72 hours after desvenlafaxine administration. Blood samples were collected in heparinized tubes. After centrifugation, separated plasma was stored at $-20^{\circ} \mathrm{C}$.

\section{Bioanalytical methodology}

To determine the plasma concentrations of desvenlafaxine in the study samples, a validated chiral liquid chromatography tandem mass spectroscopy assay was used by Advion Biosciences, with ${ }^{13} \mathrm{C}_{6}$ desvenlafaxine racemate as an internal standard. A Sciex API 3000 mass spectrometer was used; the interface was a Turbo Ionspray ${ }^{\circledR}$; other elements of the system included a LC-10AD pump, an SCL-10A pump controller, a PE series 200 autosampler, and an Astec Chirobiotic $V^{\mathbb{B}}$ column $(4.6 \mathrm{~cm} \times 100 \mathrm{~mm})$. Parallel chromatography was utilized to analyze study samples.

Nine different standard concentrations were used for the calibration curve. A single calibration curve was run with each batch; the calibration curves for all accepted runs had $\mathrm{r}^{2}$ values of 0.9949 and 0.9954 or better for (S)- and (R)-desvenlafaxine, respectively.

A solid phase extraction (SPE) procedure was performed on the plasma samples, which were analyzed and run in parallel with the calibration standards and quality control samples. Samples and quality control samples $(250 \mu \mathrm{L}$ each) were aliquoted into tomtec tubes. The Isolute $\mathrm{C}_{8} \mathrm{SPE}$ extraction block was conditioned with $1.0 \mathrm{ml}$ of methanol, then equilibrated with $1.0 \mathrm{ml}$ of water. The samples were mixed, then loaded onto the SPE block and aspirated through the cartridge at a flow rate of $1 \mathrm{ml} / \mathrm{min}$. The SPE block was washed with $1.0 \mathrm{ml}$ of water, then with $1.0 \mathrm{ml}$ 50:50 methanol:water. A full vacuum was applied, a 96-well collection block was placed into the vacuum manifold, and the analytes were eluted off the collection block with 
$0.500 \mathrm{ml}$ of methanol. Full vacuum was then applied and the collection block removed. The elute was evaporated to dryness under nitrogen at $35^{\circ} \mathrm{C}$ and the extracts were reconstituted in $200 \mu \mathrm{L} 25: 75$ isopropanol: $10 \mathrm{~m}$ ammonium acetate, $\mathrm{pH}$ 4.2. A cover film was then applied, the samples were vortexed for 1 minute, then centrifuged at $3000 \mathrm{rpm}$ for 3 minutes.

High-performance liquid chromatography was performed under isocratic conditions. The mobile phase was $25: 75$ isopropanol: $2 \mathrm{mM}$ ammonium acetate, $\mathrm{pH} 4.5$. To achieve sufficient retention and resolution, the $\mathrm{pH}$ of the aqueous component of the mobile phase was adjusted upward to ensure that the peaks eluted during the prescribed retention time windows of 6.0 minutes ( \pm 1 minute) for (S)-desvenlafaxine and 7.0 minutes ( \pm 1 minute) for $(\mathrm{R})$-desvenlafaxine. The injection volume was $10 \mu \mathrm{L}$, the flow rate was $1 \mathrm{ml} / \mathrm{min}$, and the pressure used was 160 bar. During the mass spectroscopy analysis, positive ions were measured in selected reaction monitoring mode; the ion masses monitored were 264.2 to 58.2 for desvenlafaxine and 270.2 to 58.2 for ${ }^{13} \mathrm{C}_{6}$ desvenlafaxine.

Data were acquired by and integrated on Applied Biosystems "Analyst" (version 1.4) software. Linear regression with $1 / \mathrm{x}^{2}$ weighting, performed using the software package Watson (version 7.0.0.01) (Thermo Electron Corporation, Waltham, MA), was used to fit the data to the calibration curves. Lower and upper limits of quantification were $2.5 \mathrm{ng} / \mathrm{ml}$ and $2000.0 \mathrm{ng} / \mathrm{ml}$, respectively, for both (S)- and (R)desvenlafaxine. For the quality control samples, precision was $7.5 \%$ or less for (S)-desvenlafaxine and $6.5 \%$ or less for (R)-desvenlafaxine, and the accuracy ranged from $-9.3 \%$ to $-6.6 \%$ for (S)-desvenlafaxine and from $-10.3 \%$ to $-4.1 \%$ for $(\mathrm{R})$-desvenlafaxine.

\section{Pharmacokinetic analysis}

A model-independent pharmacokinetic method of analysis was used to analyze plasma concentrations of (R)-, (S)-, and racemic mixture $(\mathrm{R}+\mathrm{S})$-enantiomers of desvenlafaxine. The concentrations of the $(\mathrm{R}+\mathrm{S})$-enantiomers of desvenlafaxine were calculated as the sum of the concentrations of the(R)- and (S)-enantiomers of desvenlafaxine. This report provides results for the racemic mixture of the $(R+S)$ enantiomers of desvenlafaxine, which will be referred to simply as desvenlafaxine.

Peak plasma concentration $\left(\mathrm{C}_{\max }\right)$ and time to peak plasma concentration $\left(\mathrm{t}_{\max }\right)$ were determined directly from the observed data. WinNonlin (Version 4.1, Pharsight Corporation, Mountain View, CA) was used for pharmacokinetic analyses. The following pharmacokinetic parameters were computed: AUC; AUC to the last quantifiable concentration at time $\mathrm{T}\left(\mathrm{AUC}_{\mathrm{T}}\right)$; $\mathrm{AUC}$ extrapolated to infinity, calculated as $\mathrm{AUC}_{\mathrm{T}}+\mathrm{CT} / \lambda_{\mathrm{z}}$, where $\lambda_{\mathrm{z}}$ is terminal-phase disposition rate constant; apparent terminal-phase elimination half-life $\left(\mathrm{t}_{1 / 2}\right)$; and apparent oral-dose clearance $(\mathrm{Cl} / \mathrm{F}) . \mathrm{AUC}_{\mathrm{T}}$ was calculated by using the log-trapezoidal rule for decreasing concentrations and the lineartrapezoidal rule for increasing concentrations. Plasma concentrations and pharmacokinetic parameters of the $(\mathrm{R})$ - and $(\mathrm{S})$-enantiomers were similar; therefore, only data for the racemic mixture are presented. Because pharmacokinetic comparisons between men and women have been previously studied and the current study design did not control for factors such as age, parameters were not evaluated separately for men and women.

\section{Safety assessment}

Safety was evaluated from spontaneously reported treatmentemergent adverse events (TEAEs), scheduled physical examinations, clinical laboratory test results, 12-lead ECGs, and vital signs. A TEAE was defined as any untoward, undesired, or unplanned clinical event that was not present when the active phase of the study began, was not part of the subject's medical history, or was present at the start of the active phase but the severity or frequency increased during the active phase. AEs were coded using the Coding Symbols for Thesaurus of Adverse Reaction Terms dictionary. Safety evaluations were reviewed throughout the study for significant deviations from normal values; changes that were of potential clinical importance based on predetermined criteria were flagged for further review by the medical monitor.

\section{Statistical analysis}

Based on data from a previous study, intrasubject variability in the desvenlafaxine AUC was estimated to be 25\% (Data on file, Pfizer Inc, formerly Wyeth Research, Collegeville, PA). The statistical power to detect a $20 \%$ difference in the AUCs of $(\mathrm{R}+\mathrm{S})$-desvenlafaxine therefore was expected to be $\geq 80 \%$ at a significance level of 0.05 with a sample size of $\geq 24$ subjects.

The pharmacokinetic parameters of desvenlafaxine were compared by dosing condition using an analysis of variance (ANOVA) for a 4-period, crossover study with the following statistical model:

$$
\mathrm{Y}_{\mathrm{ijkl}}=\mu+\mathrm{SEQ}_{\mathrm{i}}+\mathrm{SUB}_{\mathrm{j}(\mathrm{i})}+\mathrm{TRT}_{\mathrm{k}}+\mathrm{PERIOD}_{1}+\varepsilon_{\mathrm{ijkl}}
$$

$\mu=$ grand mean; $\mathrm{SEQ}_{\mathrm{i}}=\mathrm{i}^{\text {th }}$ sequence, $\mathrm{I}=1,2,3,4 ; \mathrm{SUB}_{\mathrm{j}(\mathrm{i})}=\mathrm{j}^{\text {th }}$ subject nested within the $i^{\text {th }}$ sequence, $j=1,2 \ldots 30 ; \mathrm{TRT}_{\mathrm{k}}=\mathrm{k}^{\text {th }}$ treatment, $\mathrm{k}=$ $1,2,3,4 ; \mathrm{PERIOD}_{1}=\mathrm{i}^{\text {th }}$ treatment period, $\mathrm{I}=1,2,3,4 ; \varepsilon_{\mathrm{ijkl}}=$ residual error.

In addition, the relative difference in log-transformed geometric means of $\mathrm{C}_{\max }, \mathrm{AUC}$, and $\mathrm{AUC}_{\mathrm{T}}$ of desvenlafaxine and their $90 \%$ confidence limits were calculated to estimate the magnitude of the difference in these parameters between each of the fed and fasting conditions. Fed conditions were considered bioequivalent to fasting if $90 \%$ confidence limits fell entirely within the bioequivalence acceptance range of $80 \%$ to $125 \%$. The WinNonlin Bioequivalence Wizard (Version 4.1, Pharsight Corporation, Mountain View, CA) was used for all statistical analyses.

\section{Results}

\section{Subjects}

Thirty-three subjects (17 men [51.5\%] and 16 women [48.5\%]) enrolled in the study. Five of the 33 subjects (15\%) withdrew from the study; 4 did so due to AEs. All 5 of these subjects were replaced (regardless of timing of withdrawal) with 5 subjects who received all 4 dosing conditions, and 33 subjects were included in the pharmacokinetic analysis. The subjects were healthy volunteers, with ages ranging from 18 to 45 years (mean 33.6 years). The subjects were predominately black $(\mathrm{n}=17,51.5 \%)$ or white $(\mathrm{n}=14,42.4 \%)$. A total of 4 subjects $(12.1 \%)$ used nonstudy medication. Concomitant medications were only allowed for treatment of an $\mathrm{AE}$, and included diphenhydramine hydrochloride, acetaminophen, vancomycin, cefazolin sodium, linezolid, fixed combination progestogens and estrogens, and ibuprofen.

\section{Pharmacokinetic parameters}

After administration of desvenlafaxine $200 \mathrm{mg}$, plasma concentrations were measurable in most subjects by 30 minutes after dosing in both fasting and fed conditions, regardless of the fat content of the meal. As shown by Figure 1, mean desvenlafaxine plasma concentration-time curves for each dietary condition did not differ 
Citation: Nichols Al, Richards LS, Behrle J, Posener JA, Paul J (2012) Effect of Food on the Pharmacokinetics of Desvenlafaxine in Healthy Subjects. J Bioequiv Availab 4: 024-029. doi:10.4172/jbb.1000106



Figure 1: Mean plasma concentrations of desvenlafaxine after a single, oral, $200 \mathrm{mg}$ dose, under fasting and fed conditions.

significantly.

Table 1 presents the descriptive statistics for the pharmacokinetic parameter values of desvenlafaxine administered under fed and fasting conditions. Table 2 provides the relative differences in means and $90 \%$ confidence intervals for $\mathrm{C}_{\max }$, $\mathrm{AUC}$, and $\mathrm{AUC}_{\mathrm{T}}$ for fed conditions in relation to fasting and the results of statistical comparisons based on the ANOVA of log-transformed data. The median $t_{\text {max }}$ was approximately 6 hours when desvenlafaxine was administered under fasting conditions and was delayed by approximately 2 to 4 hours when administered after low-fat, medium-fat, and high-fat meals. The delay in $t_{\max }$ under fed conditions resulted in peak concentrations for desvenlafaxine occurring 8 to 10 hours after administration.

Mean $\mathrm{C}_{\max }$ values for low-fat and medium-fat meals showed minor increases compared with fasting conditions. Based on bioequivalence criteria ( $90 \%$ confidence limits within $80 \%$ to $125 \%$ ), $\mathrm{C}_{\max }$ values were not significantly different for low-fat and medium-fat meal conditions compared with administration of desvenlafaxine under fasting conditions. In contrast, $\mathrm{C}_{\max }$ values after a high-fat meal were $16 \%$ higher compared with $\mathrm{C}_{\max }$ values under fasting conditions, and the $90 \%$ confidence interval ( $107.8 \%$ to $125.05 \%)$ marginally exceeded the upper limit of $125 \%$, but this minor increase was not considered clinically relevant.

No clear trend was observed for desvenlafaxine AUC and $\mathrm{AUC}_{\mathrm{T}}$ values to increase or decrease as fat content in meals increased. AUC values for desvenlafaxine under the low-fat, medium-fat, and high-fat meal conditions met bioequivalence test criteria ( $90 \%$ confidence limits entirely within $80 \%$ to $125 \%$ ) and were not statistically significantly different from AUC values under fasting conditions.

Other pharmacokinetic parameters of desvenlafaxine, such as $\mathrm{Cl} / \mathrm{F}$ and $t_{1 / 2}$, were not altered by food intake.

\section{Safety parameters}

Overall safety and tolerability observations were consistent with the known safety profile of desvenlafaxine from previous short-term and long-term studies in patients with MDD and the approved US product labeling. There were no new safety signals observed in the current study.

The majority of patients $(90.9 \%)$ reported one or more TEAE However, most TEAEs were mild or moderate in severity, and the frequency of TEAEs was similar across all treatment groups. The most common TEAEs included nausea, asthenia, headache, dizziness, and somnolence. Two subjects withdrew due to AEs thought to be related to administration of desvenlafaxine, namely nausea and pruritus, which weremoderateand mild in severity, respectively. One subjectexperienced a serious $\mathrm{AE}$, cellulitis secondary to an intravenous site infection prior to administering the fourth dose of desvenlafaxine, which led to study withdrawal; this was considered unrelated to desvenlafaxine treatment.

\begin{tabular}{|c|c|c|c|c|}
\hline \multirow{3}{*}{ Parameter } & \multicolumn{4}{|c|}{ Dosing Condition } \\
\hline & \multirow{2}{*}{ Fasting } & \multicolumn{3}{|c|}{ Fed } \\
\hline & & Low-fat meal ${ }^{*}$ & Medium-fat meal & High-fat meal \\
\hline $\mathrm{C}_{\max }(\mathrm{ng} / \mathrm{ml})$ & $\begin{array}{c}433 \pm 111(26) \\
{[419]}\end{array}$ & $\begin{array}{c}471 \pm 117(25) \\
{[455]}\end{array}$ & $\begin{array}{c}463 \pm 118(26) \\
{[447]}\end{array}$ & $\begin{array}{c}500 \pm 121(24) \\
{[483]}\end{array}$ \\
\hline$t_{\max }(h)^{\dagger}$ & $6.00(4.00,24.00)$ & $10.00(4.00,16.00)$ & $8.00(4.00,16.00)$ & $8.00(6.00,12.00)$ \\
\hline$t_{1 / 2}(h)$ & $\begin{array}{c}10.73 \pm 1.69(16) \\
{[10.60]^{\ddagger}}\end{array}$ & $\begin{array}{c}10.64 \pm 1.74(16) \\
{[10.49]}\end{array}$ & $\begin{array}{c}11.04 \pm 4.68(42) \\
{[10.53]}\end{array}$ & $\begin{array}{c}10.39 \pm 2.13(21) \\
{[10.18]}\end{array}$ \\
\hline $\mathrm{AUC}_{\mathrm{T}}(\mathrm{ng} \mathrm{h} / \mathrm{ml})$ & $\begin{array}{c}11019 \pm 2863(26) \\
{[10541]^{\ddagger}}\end{array}$ & $\begin{array}{c}10797 \pm 2496(23) \\
{[10434]}\end{array}$ & $\begin{array}{c}11200 \pm 2346(21) \\
{[10907]}\end{array}$ & $\begin{array}{c}10507 \pm 2609(25) \\
{[10096]}\end{array}$ \\
\hline AUC (ng'h/ml) & $\begin{array}{c}11245 \pm 2936(26) \\
{[10759]^{\ddagger}}\end{array}$ & $\begin{array}{c}10995 \pm 2558(23) \\
{[10624]}\end{array}$ & $\begin{array}{c}11496 \pm 2562(22) \\
{[11170]}\end{array}$ & $\begin{array}{c}10683 \pm 2703(25) \\
{[10259]}\end{array}$ \\
\hline $\mathrm{Cl} / \mathrm{F}(\mathrm{L} / \mathrm{h})$ & $\begin{array}{c}19.90 \pm 9.91(50) \\
{[18.59]^{\ddagger}}\end{array}$ & $\begin{array}{c}19.81 \pm 8.32(42) \\
{[18.83]}\end{array}$ & $\begin{array}{c}18.6 \pm 6.38(34) \\
{[17.90]}\end{array}$ & $\begin{array}{c}20.68 \pm 9.18(44) \\
{[19.50]}\end{array}$ \\
\hline $\mathrm{Cl} / \mathrm{F}(\mathrm{L} / \mathrm{h} / \mathrm{kg})$ & $\begin{array}{c}0.27 \pm 0.14(50) \\
{[0.25]^{\ddagger}}\end{array}$ & $\begin{array}{c}0.27 \pm 0.10(39) \\
{[0.25]}\end{array}$ & $\begin{array}{c}0.26 \pm 0.08(31) \\
{[0.25]}\end{array}$ & $\begin{array}{c}0.28 \pm 0.12(43) \\
{[0.26]}\end{array}$ \\
\hline
\end{tabular}

AUC: Area Under the Concentration-Versus-Time Curve; AUC $\mathrm{T}_{\mathrm{T}}$ AUC to the Last Quantifiable Concentration at Time T; Cl/F: Apparent Oral-dose Clearance; $\mathrm{C}_{\text {max }}$ : Peak Plasma Concentration; CV: Coefficient of Variation; $t_{1 / 2}$ : Apparent Terminal-phase Elimination Half-life; $t_{\max }:$ Time to $C_{\max }$ :

"One subject in the low-fat treatment group was excluded.

${ }^{\dagger}$ Median (minimum, maximum) for $\mathrm{t}_{\max }$

¥One subject in the fasting treatment group was excluded

Table 1: Plasma Pharmacokinetic Parameters Following a Single, Oral, 200 mg Dose of Desvenlafaxine Administered Under Fasting and Fed Dosing Conditions: Mean \pm SD $(C V \%)$, [Geometric Mean]. 


\begin{tabular}{|c|c|c|c|c|c|}
\hline \multirow{2}{*}{ Parameter } & \multicolumn{3}{|c|}{$\begin{array}{l}\text { Relative difference* } \\
\text { (90\% confidence interval) }\end{array}$} & \multicolumn{2}{|c|}{$\begin{array}{c}\text { 4-Period crossover analysis of variance } \\
\text { of log-transformed data }\end{array}$} \\
\hline & Low-fat meal & Medium-fat meal & High-fat meal & Effect & $P$-value \\
\hline $\mathrm{C}_{\max }(\mathrm{ng} / \mathrm{ml})^{\dagger}$ & $\begin{array}{c}109 \% \\
(101.1 \%-117.2 \%)\end{array}$ & $\begin{array}{c}106 \% \\
(98.6 \%-114.5 \%)\end{array}$ & $\begin{array}{c}116 \% \\
(107.8 \%-125.05 \%)\end{array}$ & $\begin{array}{l}\text { Sequence } \\
\text { Treatment } \\
\text { Period }\end{array}$ & $\begin{array}{l}0.077 \\
0.013 \\
0.860\end{array}$ \\
\hline $\mathrm{AUC}\left(\mathrm{ng}^{\star} \mathrm{h} / \mathrm{ml}\right)^{\ddagger}$ & $\begin{array}{c}99 \% \\
(90.9 \%-108.3 \%)\end{array}$ & $\begin{array}{c}105 \% \\
(96.4 \%-114.9 \%)\end{array}$ & $\begin{array}{c}96 \% \\
(88.2 \%-105.1 \%)\end{array}$ & $\begin{array}{l}\text { Sequence } \\
\text { Treatment } \\
\text { Period }\end{array}$ & $\begin{array}{l}0.282 \\
0.411 \\
0.354\end{array}$ \\
\hline $\mathrm{AUC}_{\mathrm{T}}\left(\mathrm{ng}^{*} \mathrm{~h} / \mathrm{ml}\right)^{\ddagger}$ & $\begin{array}{c}99 \% \\
(91.1 \%-108.6 \%)\end{array}$ & $\begin{array}{c}105 \% \\
(95.9 \%-114.4 \%)\end{array}$ & $\begin{array}{c}97 \% \\
(88.5 \%-105.6 \%)\end{array}$ & $\begin{array}{l}\text { Sequence } \\
\text { Treatment } \\
\text { Period }\end{array}$ & $\begin{array}{l}0.228 \\
0.512 \\
0.370\end{array}$ \\
\hline
\end{tabular}

AUC: Area Under the Plasma Concentration-Versus-Time Curve; $\mathrm{AUC}_{\mathrm{T}}$ : AUC to the Last Quantifiable Concentration at Time T; $\mathrm{C}_{\max }$ : Peak Plasma Concentration. ${ }^{*}$ Relative difference in log-transformed geometric means for fed condition relative to fasting condition.

tOne subject in the low-fat treatment group was excluded.

‡One subject in the low-fat treatment group and one subject in the fasting treatment group were excluded.

Table 2: Statistical Comparisons of Pharmacokinetic Parameters Following a Single, Oral, 200 mg Dose of Desvenlafaxine Administered Under Fed Dosing Conditions Relative to Fasting Condition.

Treatment for this AE included administration of vancomycin. Eight subjects had at least one laboratory test that met the criterion for potential clinical importance during the desvenlafaxine administration period. Nine subjects had potentially clinically important vital sign changes; of these 9, each had low supine systolic blood pressure and/ or diastolic blood pressure on 1 or 2 occasions. One of these subjects experienced a single, asymptomatic, 30-bpm increase in pulse upon standing. The potentially clinically important vital sign changes were transient with minimal changes from baseline and were not considered clinically important by the study investigator.

\section{Discussion}

Recognition of the potential for food-drug interactions is significant because of the negative clinical implications that can result from altered pharmacokinetic and pharmacodynamic properties of certain drugs. Pharmacokinetic reactions, in which food affects the absorption, distribution, metabolism, or elimination of a drug, are the most commonly observed food-drug interactions [16]. For example, concomitant intake of food can affect the rate and extent of drug absorption from the gastrointestinal tract, resulting in reduced, delayed, increased, or accelerated drug absorption [17]. In addition, the influence of food is largely dependent on the pharmaceutical formulation of a specific drug, such that food intake with some extended-release drug formulations can substantially alter the release of the drug, which ultimately alters the drug absorption time profile and, thus, the overall clinical effect [17].

The potential for food-drug interactions following administration of psychotropic medications is significant, as many of these agents are metabolized via the CYP450 enzyme system. Coadministration of antidepressant medications processed predominantly via the CYP3A4 metabolic pathway (eg, citalopram, escitalopram, fluvoxamine, paroxetine) with grapefruit juice, star fruit, or Valencia oranges can result in altered pharmacokinetic effects because of the welldocumented inhibition of these foods on the CYP3A4 system [18]. Another potential mechanism for food-drug interactions is related to alterations in drug absorption through modifications in the rate and/ or extent of absorption from the gastrointestinal tract. Delays in drug absorption or 'dose dumping' of sustained release formulations can have serious effects for safety and efficacy of a medication [19].

In the present study, we evaluated the effect of food on the pharmacokinetic profile of desvenlafaxine. Although there was not a clear progression for $\mathrm{C}_{\max }$ and AUC values to increase from fasted to the highest fat meal content treatment, there was an increase in values when comparing the high-fat meal treatment to fasting conditions. Time to maximum concentration was numerically greater with fed conditions, though the effect did not increase with varying fat content. This trend for a delayed $t_{\max }$ would be consistent with small decreases in the rate of desvenlafaxine drug absorption in the presence of food. However, it is important to note that there was considerable overlap in the variability of these parameters among the treatment groups, and the magnitude of differences was not considered to be of clinical consequence. Overall, the results demonstrate that food intake does not significantly alter exposure to desvenlafaxine and does not need to be considered when administering desvenlafaxine. The pharmacokinetic profile described here, under fasting and fed conditions, supports the once-daily administration of desvenlafaxine. A single, oral, $200 \mathrm{mg}$ dose of desvenlafaxine-4 times the recommended daily therapeutic dose of $50 \mathrm{mg}$ for MDD (in the United States) - was generally well tolerated under fasting and fed conditions in healthy adults. Given the similarity of the lower dose strengths of desvenlafaxine, the effects of fat content in meals on desvenlafaxine pharmacokinetics observed for a 200-mg dose are considered applicable to lower dose strengths of 50 and $100 \mathrm{mg}$.

The safety and tolerability profile was consistent with prior experience with similar doses in short-term and long-term studies in patients with MDD and with the safety information summarized in the approved product labeling, with no new safety signals observed in the current study. Studies investigating desvenlafaxine at lower doses (ie, 50 and $100 \mathrm{mg} / \mathrm{d}$ ) have demonstrated better tolerability and lower occurrences of AEs [14,15,20].

These findings are consistent with studies of venlafaxine that showed food did not affect the pharmacokinetics of venlafaxine or desvenlafaxine administered with once-daily dosing [13,21]. In addition, as desvenlafaxine demonstrates linear pharmacokinetics (ie, multiple-dose pharmacokinetics of desvenlafaxine are predictable from single-dose pharmacokinetics) [2], it is expected that observations in the present study related to pharmacokinetic behavior in the presence of food are applicable to chronic desvenlafaxine administration. 
Citation: Nichols Al, Richards LS, Behrle J, Posener JA, Paul J (2012) Effect of Food on the Pharmacokinetics of Desvenlafaxine in Healthy Subjects. J Bioequiv Availab 4: 024-029. doi:10.4172/jbb.1000106

\section{Study limitations}

Conclusions related to safety and tolerability in the current study are limited by the use of healthy subjects receiving a single, oral dose of desvenlafaxine and the lack of a placebo control.

\section{Conclusions}

While the rate of availability was slowed under fed conditions, exposure to a single dose of desvenlafaxine $200 \mathrm{mg}$ was largely comparable between fasting and fed conditions. Desvenlafaxine was generally well tolerated. These findings indicate that desvenlafaxine can be administered with or without food.

\section{Acknowledgements}

This study was sponsored by Wyeth, which was acquired by Pfizer Inc in October 2009. Medical writing support for this manuscript was provided by Callie Grimes, $\mathrm{PhD}$, and medical editing support was provided by Lorraine M. Sweeney, BA, and Jennifer B. Karpinski, BA,all of Embryon LLC. A Division of Advanced Health Media, LLC, and was funded by Pfizer Inc. Dr. Nichols is an employee of Pfizer Inc, formerly Wyeth Research. Ms. Richards, Ms. Behrle, Dr. Posener, and Dr. Paul were employees of Wyeth Research at the time this study was conducted.

\section{References}

1. Muth EA, Moyer JA, Haskins JT, Andree TH, Husbands GEM (1991) Biochemical, neurophysiological, and behavioral effects of Wy-45,233 and other identified metabolites of the antidepressant venlafaxine. Drug Dev Res 23: 191-199.

2. Sproule BA, Hazra M, Pollock BG (2008) Desvenlafaxine succinate for major depressive disorder. Drugs Today (Barc) 44: 475-487.

3. Bymaster FP, Dreshfield-Ahmad LJ, Threlkeld PG, Shaw JL, Thompson L, et al. (2001) Comparative affinity of duloxetine and venlafaxine for serotonin and norepinephrine transporters in vitro and in vivo, human serotonin receptor subtypes, and other neuronal receptors. Neuropsychopharmacology 25: 871 880 .

4. Deecher DC, Beyer CE, Johnston G, Bray J, Shah S, et al. (2006) Desvenlafaxine succinate: a new serotonin and norepinephrine reuptake inhibitor. J Pharmacol Exp Ther 318: 657-665

5. Nichols Al, Richards LS, Behrle JA, Posener JA, McGrory SB, et al. (2011) The pharmacokinetics and safety of desvenlafaxine in subjects with chronic renal impairment. Int J Clin Pharmacol Ther 49: 3-13.

6. Nichols Al, Fatato P, Shenouda M, Paul J, Isler JA, et al. (2009) The effects of desvenlafaxine and paroxetine on the pharmacokinetics of the cytochrome P450 2D6 substrate desipramine in healthy adults. J Clin Pharmacol 49: 219228.

7. Oganesian A, Shilling AD, Young-Sciame R, Tran J, Watanyar A, et al. (2009) Desvenlafaxine and venlafaxine exert minimal in vitro inhibition of human cytochrome P450 and P-glycoprotein activities. Psychopharmacol Bull 42: 4763.
8. Patroneva A, Connolly SM, Fatato P, Pedersen R, Jiang Q, et al. (2008) An assessment of drug-drug interactions: the effect of desvenlafaxine and duloxetine on the pharmacokinetics of the CYP2D6 probe desipramine in healthy subjects. Drug Metab Dispos 36: 2484-2491.

9. Preskorn S, Patroneva A, Silman H, Jiang Q, Isler JA, et al. (2009) Comparison of the pharmacokinetics of venlafaxine extended release and desvenlafaxine in extensive and poor cytochrome P450 2D6 metabolizers. J Clin Psychopharmacol 29: 39-43.

10. Nichols Al, Focht K, Jiang Q, Preskorn SH, Kane CP (2011) Pharmacokinetics of venlafaxine extended release $75 \mathrm{mg}$ and desvenlafaxine $50 \mathrm{mg}$ in healthy CYP2D6 extensive and poor metabolizers: a randomized, open-label, twoperiod, parallel-group, crossover study. Clin Drug Investig 31: 155-167.

11. Nichols AI, Behrle JA, Richards LS, Parker VD, Posener JA, et al. (2012) The absolute bioavailability of desvenlafaxine in healthy subjects. $J$ Bioequiv Bioavail 4: 018-023.

12. Behrle JA, Nichols AI, McGrory SB, Raible D (2005) An open-label, randomized single-dose, dose-proportionality study of oral doses of a sustained-release formulation of desvenlafaxine succinate in healthy subjects. Clin Pharmacol Ther 77: P82.

13. Parks V, Patat A, Behrle J, Parker V, Decours J (2005) Safety, pharmacokinetics (PK) and pharmacodynamics (PD) of ascending single doses of desvenlafaxine (DVS-233 SR) in healthy subjects. Clin Pharmacol Ther 77: P28.

14. Boyer P, Montgomery S, Lepola U, Germain JM, Brisard C, et al. (2008) Efficacy, safety, and tolerability of fixed-dose desvenlafaxine 50 and 100 $\mathrm{mg} /$ day for major depressive disorder in a placebo-controlled trial. Int Clin Psychopharmacol 23: 243-253.

15. Liebowitz MR, Manley AL, Padmanabhan SK, Ganguly R, Tummala R, et al (2008) Efficacy, safety, and tolerability of desvenlafaxine $50 \mathrm{mg} /$ day and 100 $\mathrm{mg} /$ day in outpatients with major depressive disorder. Curr Med Res Opin 24 1877-1890.

16. Schmidt LE, Dalhoff K (2002) Food-drug interactions. Drugs 62: 1481-1502.

17. Singh BN (1999) Effects of food on clinical pharmacokinetics. Clin Pharmacokinet 37: 213-255.

18. Ereshefsky L (2009) Drug-drug interactions with the use of psychotropic medications. CNS Spectr 14: 1-8.

19. Fleisher D, Li C, Zhou Y, Pao LH, Karim A (1999) Drug, meal, and formulation interactions influencing drug absorption after oral administration. Clinical Implications. Clin Pharmacokinet 36: 233-254.

20. Clayton AH, Kornstein SG, Rosas G, Guico-Pabia C, Tourian KA (2009) An integrated analysis of the safety and tolerability of desvenlafaxine compared with placebo in the treatment of major depressive disorder. CNS Spectr 14: 183-195.

21. Troy SM, Turner MB, Unruh M, Parker VD, Chiang ST (1997) Pharmacokinetic and pharmacodynamic evaluation of the potential drug interaction between venlafaxine and ethanol. J Clin Pharmacol 37: 1073-1081. 